\title{
A RARE CASE OF PANCREATIC PARAGANGLIOMA: HISTOLOGICAL AND IMMUNOHISTOCHEMICAL ASPECTS
}

Mahendra Singh 1 , Adrija Pathak², Rachita Gulati ${ }^{3}$

\section{HOW TO CITE THIS ARTICLE:}

Mahendra Singh, Adrija Pathak, Rachita Gulati. "A Rare Case of Pancreatic Paraganglioma: Histological and Immuno-histochemical Aspects". Journal of Evolution of Medical and Dental Sciences 2015; Vol. 4, Issue 09, January 29; Page: 1563-1568, DOI: 10.14260/jemds/2015/218

ABSTRACT: INTRODUCTION: Paragangliomas, are rare neuroendocrine neoplasms that are derived from the paraganglia. Extra-adrenal paraganglioma arising within the pancreas is an extremely rare entity. CASE REPORT: Here we present a case of 72 year male patient with the complaints of abdominal distension and ultrasound showing a large multicystic mass measuring 12 x $8.5 \mathrm{~cm}$ extending from the tail of the pancreas to the pelvis. DISCUSSION: The diagnosis was made as a rare case of pancreatic paraganglioma on the basis of histological examination and immune histochemistry.

KEYWORDS: Paraganglioma, zell ballen, chromogranin.

INTRODUCTION: Paragangliomas, also known as extra-adrenal pheochromocytomas, are rare neuroendocrine neoplasms that are derived from the paraganglia, a diffuse neuroendocrine system dispersed from the skull base to the pelvic floor. They are found in many tissues such as the adrenal medulla, the carotid bodies, the organs of Zuckerkandl, and the paraganglia of the sympathetic and parasympathetic neurons. ${ }^{[1]}$ Extra-adrenal paraganglioma arising within the pancreas is an extremely rare entity with only 12 cases reported in the literature.[2]

CASE REPORT: A 72 year male patient was admitted in our hospital with the complaints of abdominal distension since 6-7 months with passage of slight mucoid like discharge with faeces. He had no other complaints of pain or bleeding per rectum or fever. His bowel movements were normal. On physical examination, lump could not be palpated. There was no hepatomegaly or splenomegaly or any lymphadenopathy. His blood picture was completely normal.

On ultrasound, it showed a large multicystic mass measuring $12 \times 8.5 \mathrm{~cm}$ extending from the tail of the pancreas to the pelvis displacing the bowel loops slightly to one side.

On gross examination the specimen was a large globular cystic tissue. The outer surface was glistening grayish brown to many haemorrhagic areas and congested blood vessels. On cut section, it was multicystic with occasional friable firm grayish brown areas. The cysts were filled with brownish fluid like material. The cyst wall thickness varied from papery thin to $0.1 \mathrm{~cm}$.

Microscopically sections from firm areas showed pancreatic tissue with serous gland and ducts. Rest of the areas showed small round cells with eccentric nuclei with salt and pepper type of chromatin and moderate to abundant amphophilic cytoplasm. These cells were forming organoid pattern or nests separated by fibrous tissue and vascular channels. This is the characteristic Zell ballen pattern paragangliomas.

Immunohistochemistry for Chromogranin was diffusely positive with punctate cytoplasmic positivity. S 100 was focally positive depending upon the number of sustentacular cells which was 


\section{CASE REPORT}

relatively less in this particular case. Cytokeratin was negative ruling out any carcinomas. Ki 67 was focal positive, less than $2 \%$ showing this tumor is benign and no malignant change is taking place.

Thus morphology, immunohistochemical features were consistent with pancreatic paraganglioma.

DISCUSSION: Pheochromocytomas are tumors that arising from chromaffin cells of the adrenal medulla. They are called paraganglioma if chromaffin-cell tumors originate from extra-adrenal sites along the sympathetic and/or the parasympathetic chain. They secrete and store catecholamines causing some symptoms (functional tumors); some of them, especially parasympathetic paragangliomas, may be non-functional. ${ }^{[3]}$ They may present at all ages with a peak incidence around 30-50 years. Clinical incidences have been estimated 1.5 per million per year in the case of paragangliomas. ${ }^{[4]}$

Pancreatic paragangliomas are generally non-functional,[5] in contrast to tumors arising from other sites, where functional activity is more common. In tumors associated with symptomatic hypertension, headache, palpitations, and sweating, catecholamine secretion is reported in $30-60 \%$ of cases. Diagnosis of retroperitoneal paraganglioma could be delayed as it usually relies on the growth of the tumor mass. It is hard to make a correct preoperative diagnosis due to absence of typical clinical symptoms in the patient. CT, MRI or ultrasonographic studies are sensitive in detecting a retroperitoneal mass and could delineate its location, outline, internal structure as well as its relationship with the surrounding organs. However, specific diagnosis for the retroperitoneal mass still relies on postoperative histopathological diagnosis. In addition, histopathological diagnosis is required to define the paraganglioma as benign or malignant tumours exhibit similar clinical diagnosis and imaging findings.[6] Malignancy is uncommon, and there is no definite histological criterion to differentiate between benign and malignant counterparts. ${ }^{[7]}$ The only absolute indication of malignancy may be distant metastasis to the organs such as liver, bone and lymph nodes. Certain histological features such as invasion; vascular and/or capsular, confluent or focal necrosis, diffuse growth or large nests, high cellularity nuclear pleomorphism and hyperchromasia are suggestive of malignancy.[8] Several possible characteristics are suggested as predictors of malignancy: tumor weight more than $80 \mathrm{~g}$ and high concentration of dopamine inside the tumor, tumor size more than 5 $\mathrm{cm}$ (75\% predictive).[3] $\mathrm{Ki}-67$ proliferative index more than $6 \%$ is most common in malignant tumors.[9]

The use of an immunohistochemical panel, in addition to routine histology, can confirm the diagnosis of a paraganglioma and can give an indication of the likely prognosis for a patient. Many immunohistochemical markers are used in diagnosis of neuroendocrine tumors: enzymes (neuron specific enolase, NSE); proteins stored in the secretory granules (chromogranin A and HISL- 19 protein); resident proteins of the presynaptic vesicles; proteins of the cytoskeleton (neurofilament); catecholamines and indolamines (epinephrine, norepinephrine, dopamine, serotonine); neuropeptides (enkephaline, VIP, corticotropine); molecules with unknown functions (PGP 9.5, myelin associated glycoprotein Leu-7).

Chromogranins are excellent indicatives of neuroendocrine differentiation. Its function is to stabilize the intra-cellular matrix of neurosecretory granules. A well differentiated tumor usually contains more granules than those with poor differentiation. The low level of chromogranin 
sensitivity is a limiting factor for its diagnostic application. NSE and chromogranin together have a sensitivity that approaches to $100 \% \cdot{ }^{[10]}$

S100 protein, a dimeric $21-\mathrm{Kd}$ protein bonding with calcium, was isolated from the nervous system. It has been identified in sustentacular cells of autonomous ganglia, adrenal medullas and extra-adrenal paraganglia. It may also be identified in sustentacular cells of neural and neuroendocrine neoplasias. It is localized in the cytoplasm and nuclei of the sustentacular cells of extra-adrenal paraganglia. In extra-adrenal paraganglioma, it has been demonstrated that all the benign tumors contain sustentacular cells, whereas these are seen to be absent in malignant tumors. ${ }^{[10]}$

The nuclear antigen AgKi67 (MiB-1) is a protein in all phases of the cell cycle except the G0 phase, and has a direct relationship with the growth rate of a cell population. It is an excellent marker for cell proliferation.[11] Our immunohistochemical analysis revealed that the tumor was positive for Chromogranin A, S-100, cytokeratin negative and Ki67 index $<2 \%$ with a characteristic zellballen pattern on histopathology, thus providing a basis for a correct diagnosis of nonchromaffin paraganglioma of the retroperitoneum in the patient arising from pancreas.

Meticulous and complete surgical removal of the tumor has been reported to associate with long-term survival. Most paragangliomas have an intact capsule with abundant blood vessels both on its surface and inside. Therefore, most paragangliomas can be removed without much difficulty if the tumor is meticulously resected.

\section{REFERENCES:}

1. Yeo H, Roman S: Pheochromocytoma and functional paraganglioma. Curr Opin Oncol 2005, 17: 13-18.

2. He J, Zhao F, Li H, Zhou K, Zhu B. Pancreatic paraganglioma: A case report of CT manifestations and literature review. Quant Imaging Med Surg, 2011.

3. Chrisoulidou A, Kaltsas G, Ilias I, Grossman AB. The diagnosis and management of malignant pheochromocytoma and paraganglioma. Endocrinology and Related Cancer 2007; 14 (September (3)): 569-85.

4. Erickson D, Kudva YC, Ebersold MJ, Thompson GB, Grant CS, Van Heerden JA, et al. Benign paragangliomas: clinical presentation and clinical outcomes in 236 patients. Journal of Clinical Endocrinology and Metabolism 2001; 86 (11): 5210-6.

5. Kim SY, Byun JH, Choi G, Yu E, Choi EK, Park SH, et al. A Case of primary paraganglioma that arose in the pancreas: The color doppler ultrasonography and dynamic CT features. Korean J Radiol 2008; 9: S18-21.

6. Tambo M, Fujimoto K, Miyake M, Hoshiyama F, Matsushita C, Hirao Y: Clinicopathological review of 46 primary retroperitoneal tumors. Int J Urol 2012, 14: 785-788.

7. Awasthi NP, Kumari N, Krishnani N, Goel A. 'Functional' paraganglioma of ureter: An unusual case. Indian J Pathol Microbiol 2011; 54: 405-6.

8. Andersen KF, Altaf R, Krarup-Hansen A, Kromann-Andersen B, Horn T, Christensen NJ, et al. Malignant pheochromocytomas and paraganglomas - the importance of a multidisciplinary approach. Cancer Treatment Reviews 2011; 37 (April (2)): 111-9. 


\section{CASE REPORT}

9. Salmenkivi K, Heinkkila P, Haglund C, Louhimo J, Arola J. Lack of histologically suspicious features, proliferative activity and p53 expression suggests benign diagnosis in pheochromocytomas. Histopathology 2003; 43: 62-71.

10. Kliewer KE, Wen DR, Cancilla PA, Cochran AJ. Paragangliomas: Assessment of prognosis by histologic, immunohistochemical, and ultrastructural techniques. Hum Pathol 1989; 20: 29-39.

11. Schwarting R. Little missed markers and Ki67. Lab Invest 1993; 68: 597-9.

Fig. 1: On gross examination, tumor measures $12 \times 8.5 \mathrm{~cm}$. It is well circumscribed and outer surface looks smooth and shiny with congested blood vessels.

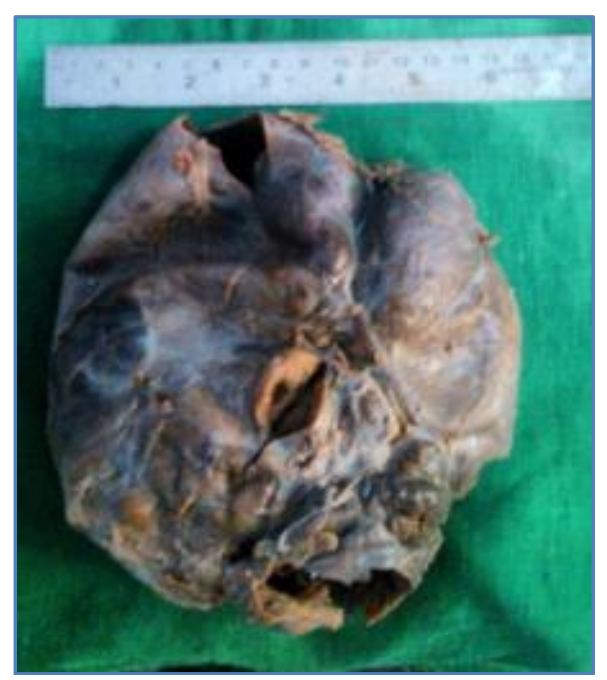

\section{Fig. 1}

Fig. 2: Cut section shows a multilocular cyst with glistening surface and areas of haemorrhage. Cyst wall is papery thin

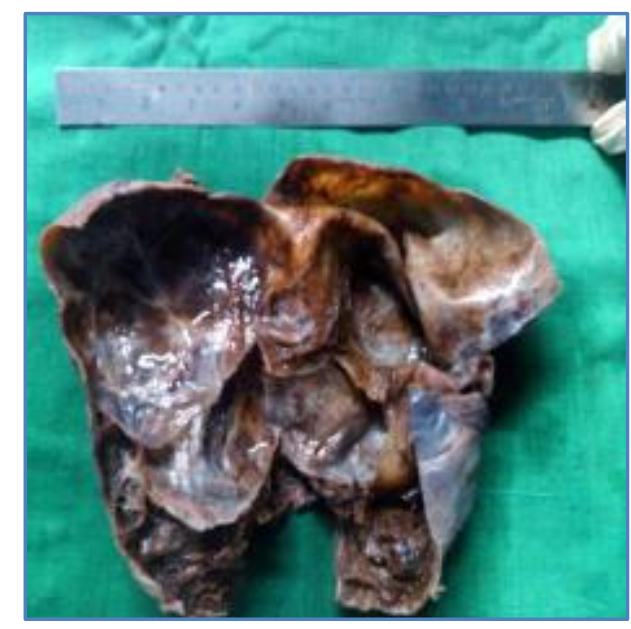

\section{Fig. 2}




\section{CASE REPORT}

Fig. 3: Low power view showing the characteristic Zell ballen pattern.

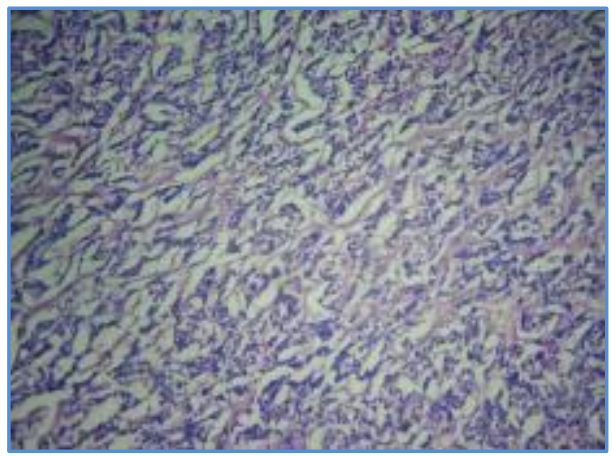

Fig. 3

Fig. 4: Microscopic view of tissue showing pancreas with serous glands and ducts.

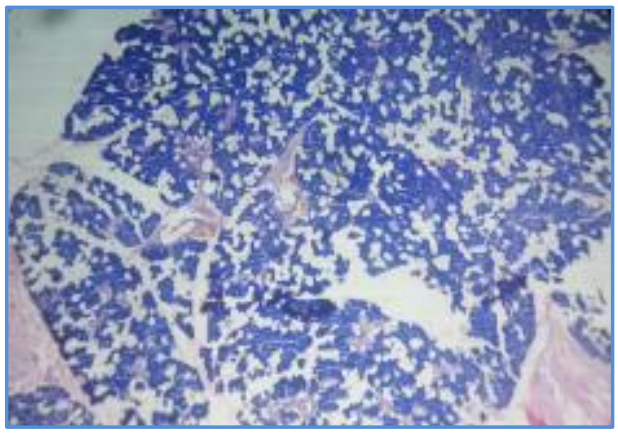

Fig. 4

Fig. 5: Immunohistochemistry showing diffuse cytoplasmic positivity for chromogranin(A), Sustentacular cells showing S100 positivity (B),Cytokeratin negativity (C) and Ki-67 showing <2\% positivity(D).

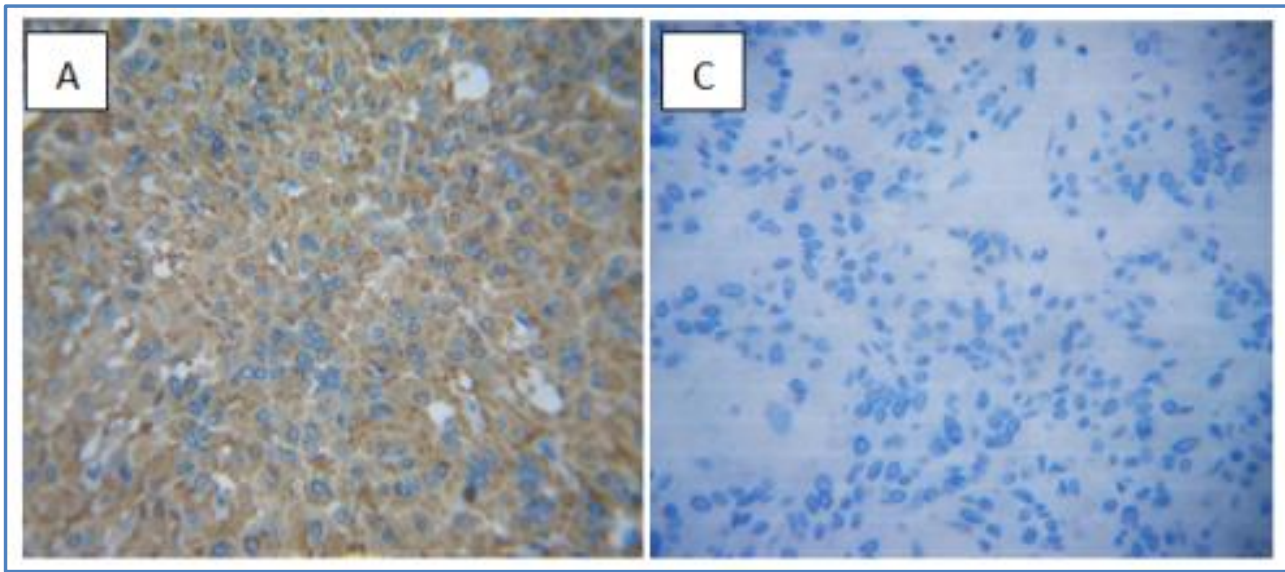

Fig. 5A

\section{Fig. 5B}




\section{CASE REPORT}

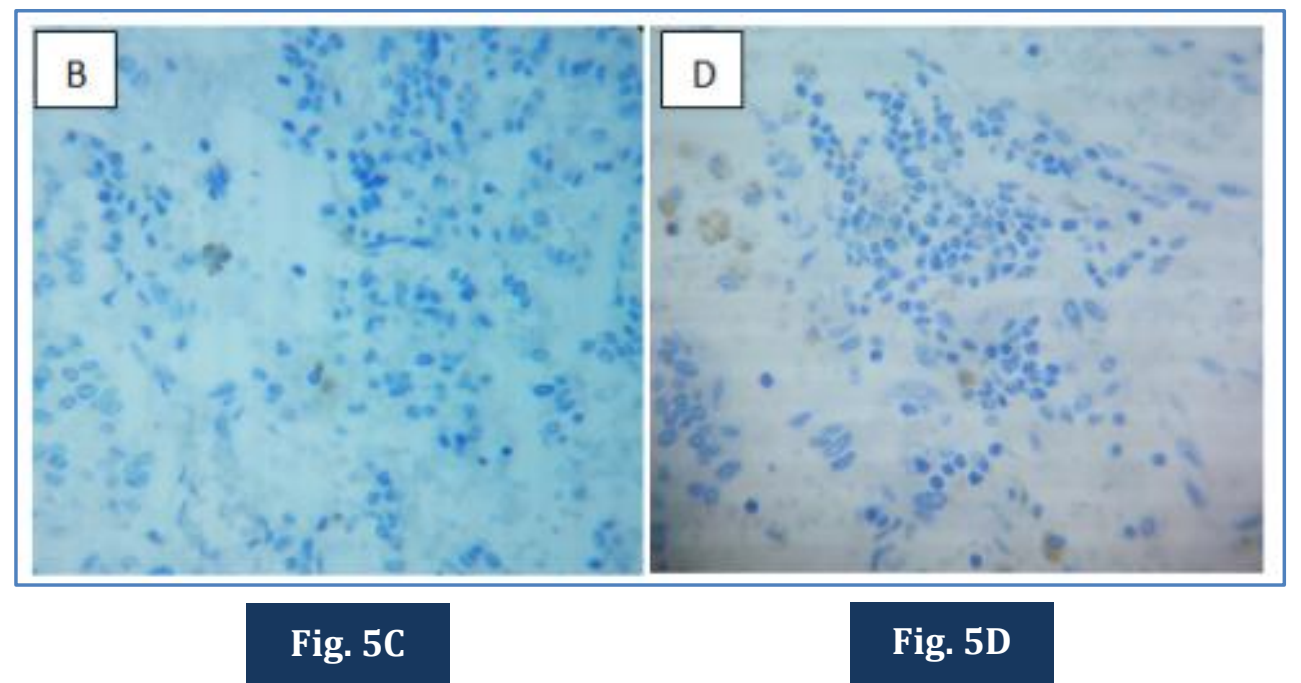

\section{AUTHORS:}

1. Mahendra Singh

2. Adrija Pathak

3. Rachita Gulati

\section{PARTICULARS OF CONTRIBUTORS:}

1. Professor \& HOD, Department of Pathology, G. S. V. M. Medical College, Kanpur.

2. Junior Resident, Department of Pathology, G. S. V. M. Medical College, Kanpur.

3. Junior Resident, Department of Pathology, G. S. V. M. Medical College, Kanpur.

\section{NAME ADDRESS EMAIL ID OF THE} CORRESPONDING AUTHOR:

Dr. Adrija Pathak,

Room No. 8,

New Married Hostel,

Halet Hospital,

Kanpur-208002.

E-mail: pathakadrija@gmail.com

Date of Submission: 01/01/2015.

Date of Peer Review: 02/01/2015.

Date of Acceptance: 19/01/2015.

Date of Publishing: 29/01/2015. 